“(C) 2018 IEEE. Personal use of this material is permitted. Permission from IEEE must be obtained for all other uses, in any current or future media, including reprinting/republishing this material for advertising or promotional purposes, creating new collective works, for resale or redistribution to servers or lists, or reuse of any copyrighted component of this work in other works." 


\title{
Modernising Asthma Management: Personalised Asthma Action Plans Using a Smartphone Application
}

\author{
Nikita Isaac, Naveena Sampath and Valerie Gay \\ Faculty of Engineering and IT \\ University of Technology Sydney \\ Broadway NSW 2007, Australia \\ Email: Valerie.Gay@uts.edu.au
}

\begin{abstract}
Asthma is a chronic disease affecting one in nine Australians. With symptoms such as coughing, wheezing and shortness of breath. asthma can significantly impact a patient's quality of life. Asthma action plans are said to be one of the most effective asthma interventions available. However, in Australia only one in five people aged 15 and over, with asthma, have a written asthma action plan. Even less of which, refer to their plan. A review of related literature and work showed a gap regarding accessibility of information on asthma action plans in a written form. In an attempt to mitigate this problem, this paper focuses on the design and development of a smartphone application. The application is currently a high-fidelity prototype designed and built using proto.io software. In addition to this conversion, the application incorporates aspects of the Internet of Things (IoT) whereby realtime data regarding environmental triggers such as temperature, humidity and pollen in surroundings, can be accessed from the application. The application ultimately aims to help asthmatics improve their health and quality of life by providing them, or their carer with the knowledge needed to better understand and manage their asthma, when and where they need it.
\end{abstract}

Keywords-Asthma management; mobile technology; smartphone application; asthma action plans; internet of things

\section{INTRODUCTION}

Asthma is a chronic disease affecting one in nine Australians [1]. As of 2014, 1.5 out of every 100, 000 deaths in Australia were due to asthma [2]. People with asthma have sensitive airways which react to environmental triggers, causing 'flare ups'. This is when muscles in the wall of airways tighten and swell, narrowing the airway itself. This, in combination with the production of mucus can block the airway to varying degrees. Resulting in symptoms such as coughing, wheezing, tightness in the chest and shortness of breath, making it extremely difficult to breathe [3].

There is no cure for asthma, even when patients feel fine, they still have asthma and flare ups can occur at any time [4]. Asthma varies in severity from mild to severe. In severe asthma, breathing difficulties can be life threatening [5].

Asthma Australia's 2015-16 annual report, stated the individual cost of having asthma as $\$ 11741$ per asthmatic, and the direct and indirect costs of asthma to the Australian economy was an estimated $\$ 28$ billion per annum. With $\$ 1.2$ billion being costs to the health system by means of prescriptions and hospitalisations. In the Australian Institute of
Health and Welfares 2014-15 report, there were 39500 hospitalisations and a totalled 419 deaths from due to asthma.

Asthma can be managed by taking an active role in its management via ongoing treatment and building a strong partnership with doctors and other health care providers [1]. Asthma action plans are said to be one of the most effective asthma interventions available. A Written asthma action plan is key to effective asthma management, because it is written by the patient, in conjunction with their doctor. Such that they can both easily recognize changes in the patient's asthma severity and provide clear instructions on how to respond. It is important to note that as a patient's asthma severity changes so should the advice on their asthma action plan. Hence regular updates are essential [3]. However, despite the recommendation that all asthmatics in Australia be given a personalised asthma action plan (PAAP), only one in five people aged 15 and over with asthma, have a written asthma action plan in Australia [2], even less of whom refer to it.

The biggest challenge with asthma control is daily adherence. Consequently, this projects goal is to increase the awareness and use of Asthma action plans as a key asthma intervention for regular control over symptoms.

In this paper section II consists of an evaluation of related work regarding the benefits and current limitations of PAAPs, the current use of smartphone applications and the Internet of Things (IoT) in health care. The overlap of these three themes leads to the design and development of a high-fidelity prototype in sections III and IV. This is followed by a discussion in section $\mathrm{V}$ and conclusion in section VI which evaluates the application for future improvements and general biases.

\section{RELATED WORK}

\section{A. Asthma Action Plan}

In the UK asthmatics without a PAAP are said to be four times more likely to be hospitalised for asthma related purposes [5]. PAAPs are noted by Gatheral et al (2017) to be fundamental in the achievement of asthma control. Asthmatics are frequently sent to the ED for reasons that could be easily handled with proper understanding of their asthma and how to treat it at home [6]. Steady increases in ED admissions for asthma causes in patients aged 14-19 years old in the UK, indicated a need for a 
clinically appropriate program to encourage effective asthma management [5]. This need can be further supported as Asthma flare-ups were the top cause for ED visits for children in the US over 2011 [7]. Consequently, Lynch et al (2016) conducted an asthma review where participants were to use a peak flow meter and an Asthma Action Plan (AAP) for two months with weekly telephone check-ups on their condition. The results showed improved consistency of exacerbations, self-reported wellbeing and reduced hospitalisations, with $75 \%$ of participants having a better understanding of their own asthma and as a result of this, with $83 \%$ of patients feeling more in control of their asthma. Overall half of the participants experienced improved asthma symptoms as a result of their reliance on their AAP.

While all these studies demonstrate how beneficial PAAPs can be to an asthmatic, PAAPs are not without current limitations. The National Health Service in Lanarkshire, Scotland and the National Service for Health Improvement in the UK, developed an adult version of the PAAP and assessed its usefulness within the local population. Smith et al (2016) conducted the study to understand the impact of a structured consultation delivered by a respiratory nurse or specialist on a patient use of a PAAP and consequently their asthma. As part of a structured annual review and educational program, 136 patients were provided with a PAAP in consultation with a respiratory nurse specialist team. Over the next 6 months, 27.2\% of participants experienced asthma exacerbations. At the 6month follow up point, $82.2 \%$ remembered getting their PAAP but only $64.9 \%$ of participants still had theirs and only $20.7 \%$ of participants had properly used their PAAP. Additionally, $43.4 \%$ of patients were not able to name even one item or task on their PAAP. These results indicate that within a short 6-month time period, one third of patients had not stuck to their PAAP and this was largely due to their reported difficulties in recalling and accessing its content. This highlights the need for constant access to the information on a PAAP in order for its continuous and effective use.

\section{B. Smartphone Application}

In the face of inconsistent and ignored face-to-face and paper-based tools evidenced by the 1 in 5 usage rate of written asthma action plans in Australia [1], apps are increasingly recognized by policy makers as a potential avenue of supporting self-management in long term conditions, such as asthma [8]. Huckvale, Car, Morrison \& Car (2012) conducted a review on the extent to which current smartphone apps suitably support people with asthma, by way of comprehensive information, evidence-based advice and compliance with best practice health principles. After performing a search in Android and iOS app stores against an inclusion and exclusion criteria, 94 English apps were found. Of which, 43 apps were sources of written information about asthma, 13 were multimedia apps and the outstanding 47 were tools supporting aspects of asthma selfmanagement. Despite this, they found no English language app that combined information and management tools, identifying that the potential complexity of these tasks highlights an opportunity to combine information and management tools. In 2015 , they conducted a review of the 2012 data, to highlight the evolution of smartphone apps for asthma, and noted that with the rapid evolution of mobile technologies, opportunities for health capabilities by means of wearable devices are emerging. Between 2011 - 2013, the number of apps for asthma doubled. Based on the previously mentioned inclusion and exclusion criteria apps from both studies were screen again and an updated analysis was performed on 147 unique asthma apps. 83\% of asthma apps available in 2013 gave details of asthma pathophysiology. But only half provided information on basic asthma management. Even less addressed self-management skills inclusive of allergen and trigger avoidance, selfmonitoring skills or how to appropriately use treatment. Furthermore, less than a third of apps addressed how to recognize signs of deterioration or failed to identify the importance of personalized treatments and goal setting.

At the same time, there is no current smartphone health or fitness app that meets the need of every patient [8]. Huckvale, Car, Morrison \& Car (2012) found 103 English apps, but none of them combined comprehensive, evidence based information with supportive tools. The review showed that paper based asthma action plans lack the interactive treatment recommendations offered by most app based plans in the review. However, asthma apps enabling the development and updating of asthma action plans or data into diaries, were noted as being in need of a new way to populate records in a timely fashion. As a result, the quality of an app may be judged differently by different patient users of the app specific to their own goals such that it stimulates sustained use. The range of app functions for asthma demonstrated in this literature review highlights the potential value of patient and professional input into health app designs.

\section{Internet of Things}

Although recent years have seen a vast improvement in medications to control asthma symptoms, asthma management still proves a challenging task as it requires an understanding of asthma causes and the consequent triggers to avoid. Both of which are multi-factorial and individualistic in nature [9]. Additionally, it is not possible for doctors to constantly monitor each patient's health with respect to their environmental triggers.

Several prototype devices and systems incorporating IoT and asthma are currently being developed. Sheth et al (2017) published a research paper outlining their work on putting together ' $\mathrm{kHealth}$ ' which is a model for the ongoing monitoring of a patients personal, public and population-based health signals. Using this information, it can send alerts regarding the severity of a patient's asthma condition to both the patient and their clinician. In a similar, yet alternative way, Bozkart (2015) and his team at The National Science Foundation (NSF) Nanosystems Engineering Research Centre for Advanced SelfPowered Systems of Integrated Sensors and Technologies (ASSIST) research centre developed a wearable sensor system composed of a wristband and chest patch which together, pull correlating information about an individual's environmental exposure and its impact on their asthma conditions. 
kHealth enables the clinician to gather information regarding their patients triggers and put together a prevention plan for the future. Furthermore, it gives patients the chance to take control of their asthma management [10]. kHealth attempts to personalise and contextualise the information it gathers using multi-modal data, to help better understand asthma control. This program is currently undergoing trials at the Dayton Children's hospital in Ohio, with 200 patients. Part of the study used the Foobot sensor to measure indoor air quality. Indoor environments vary significantly depending on how they are being used. Indoor air conditions change while performing certain tasks or activities such as cooking, vacuuming or smoking. The study showed that combinations of these activities had a great effect on asthma in children. Consequently, a continuous monitoring system was set up to obverse and understand the indoor air quality of asthma patients. Data was collected from 7 environments over 15 days and was examined to successfully determine that high concentrations of particulate matter, carbon dioxide and volatile organic compounds during cooking and smoking events, do have an impact on children with asthma. Using a control of when no event was taking place, they detected cooking with an error rate of $11 \%$, smoking with an error rate of $1 \%$, obtaining an overall $95.7 \%$ percent classification accuracy across all three events.

\section{DESIGN OF APPLICATION}

Using the prototype software and design tools of Proto.io, a high-fidelity prototype of the proposed asthma action plan app was designed. In order to ensure further consistency, the graphics and terminology used in the National Asthma Council Australia's written asthma action plan were kept and adapted to the smartphone applications digital environment and needs. Figure 3.1. is a flowchart outlining the high-level storyboard of events within the app. At the end of each page there is an option to return to the homepage. Figure 3.2 is a detailed flowchart outlining the steps taken for initial set-up of the application. Once the user reaches the IoT tab, depending on what information they desire, they will be directed to either AccuWeather for temperature and humidity or WeatherZone for pollen.

A baseline survey of 32 patients was conducted regarding the future use of a PAAP smartphone application. The establishment of patients need and desire for the PAAP smartphone application lead to the end-user ranking of app features, cross-referenced with clinical design requirements already in place by the National Asthma Council Australia, plus the addition of an IoT feature.

This feedback revealed that features such as knowing when to seek urgent medical help, and information regarding call-toaction responses and management of increased severity were of the most importance to users. However, all features were found to be at least somewhat important, with majority of participants indicting that all features were 'important' or 'very important'.
Consequently, all features were incorporated into the PAAP smartphone application, with emphasised important put on the top three features as chosen by participants.

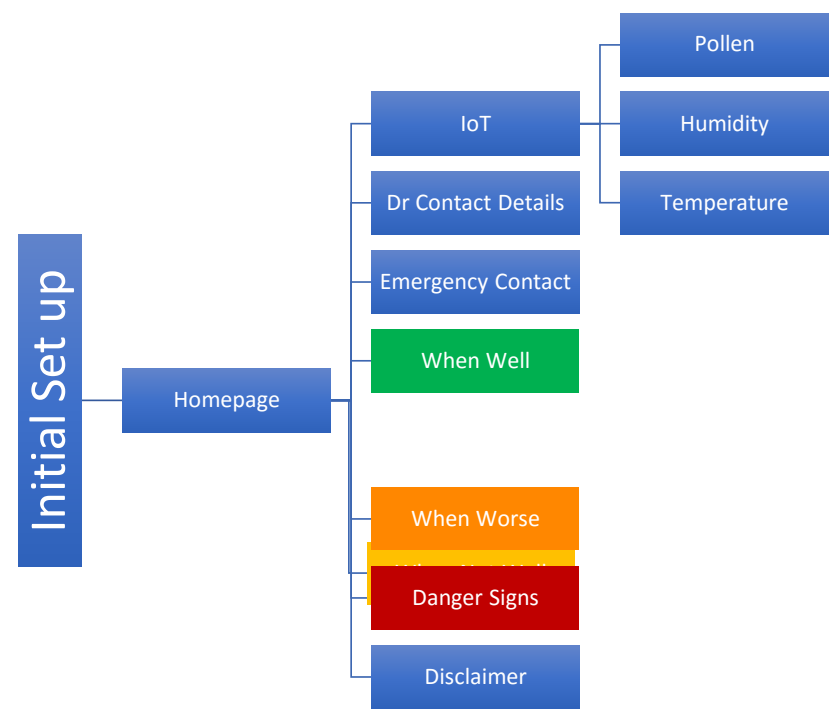

Figure 3.1 High level flowchart of PAAP app
Figure 3.2 Detailed flowchart of initial set up

\section{RESULTS}

In addition to assessing the current contextual use of PAAPs, the Asthma Action Plan Survey also addressed the feasibility of an asthma action plan smartphone application in terms of user perspective, requirements and desire for such a platform.

When asked about whether or not participants think an app version of hand-written AAPs would increase their use $87.5 \%$ said yes. Additionally, when asked if they would be interested in using said app, $93.8 \%$ said yes. Of the 32 responses to the survey, $68.8 \%$ do not currently use any type of health or fitness tracking application. The remaining $31.1 \%$ that do, list their 
ease of use, convenience and monitoring capabilities as the three most common reasons for using these health applications. Top- 5 valued features as ranked my participents were:

- Danger signs - when and how to seek urgent medical help

- Contain information on what action to take in response to exacerbations

- Managing increased severity

- Treating exacerbations

- Contain information for users to recognise exacerbations

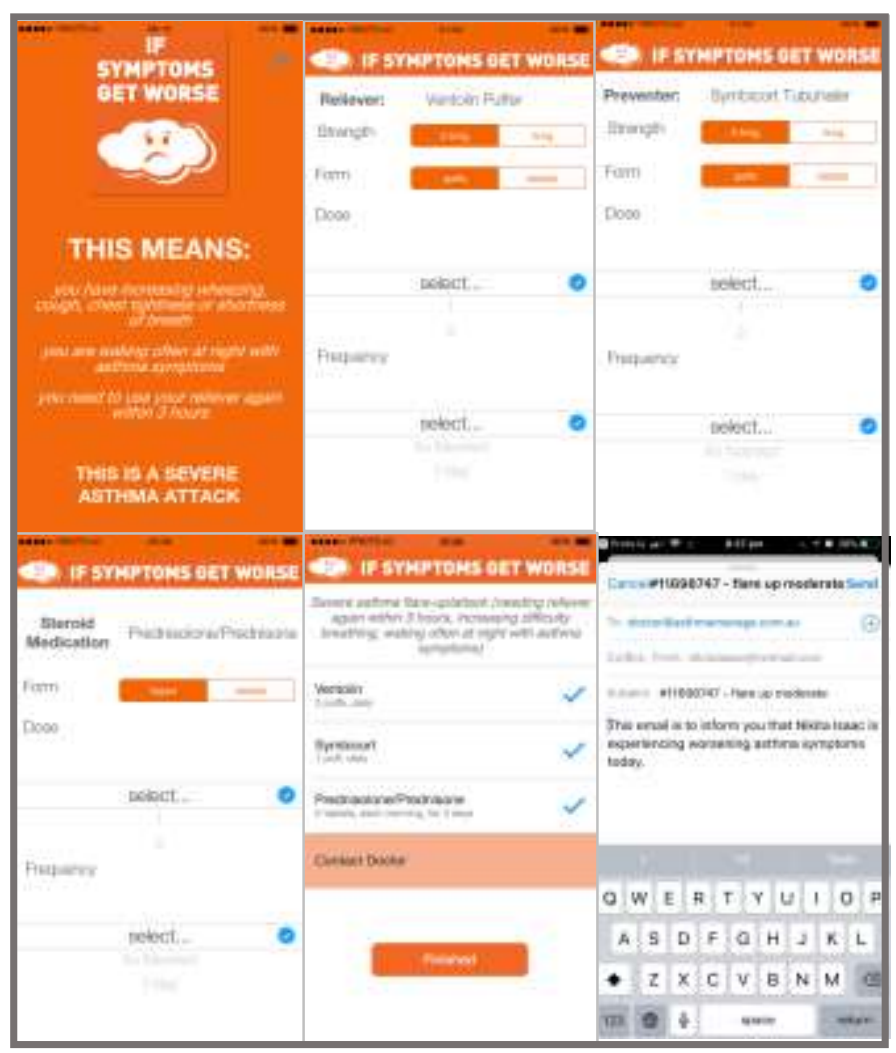

Figure 4.1 Six stages of 'Orange - if symptoms get worse' within application

With all these factors in mind, a high-fidelity prototype was made. The app functions fully as a prototype allowing the user to enter the details of themselves and their doctor, followed by their doctors recommended treatments for each stage of exacerbations. The application explicitly outlines how to identify the severity of a flare up. It has an integrated checklist system making it easy to use and track progress of treatment at the time. Here the 'Orange - If symptoms get worse' level is used to demonstrate how the application will work and how key features from the survey were integrated into the app.

Taking into consideration feedback from the forms and the requirements on the existing written PAAP forms, when a patent is experiencing worsening symptoms it is advised that they contact their physician to let them know. Accordingly, as shown in Figure 4.1, when in the 'Orange - If symptoms get worse' section of this application, rotating the device automatically sends your entered physician an email notification to let them know.

\section{DISCUSSION}

It is important to note that survey data was collected from online asthma help-groups. Patients therefore, are likely to have a preference for technology as a solution for their asthma. Consequently, a key improvement to be made is to increase the number of patients that complete the forms. The responses of 32 patients alone are not sufficient to make definitive conclusions. This would also even out the potential technology bias. This design of the smartphone application is largely focused on patient's requirements and incorporates some aspects of the carers perspective. However, in order to gain a complete and holistic application, significantly more input from carers and more importantly, doctors will be required.

Another aspect to be considered in further detail for the future is the security of personal health data. Especially with the use of IoT there will be need to ensure the security of this information. One way might be through the use on encryptions that only patient, carer and doctor are aware of.

As previously mentioned, the current PAAP smartphone application is a high-fidelity prototype. As a result, it has present limitations and requires improvements by means of continued development of the application, so it is fully functional. More specifically, this includes improvements regarding user interface, to be more attuned with natural hand movements and gestures made when using smartphone applications. As well as having more embedded IoT connections within the app. Rather than selecting the IoT tab and having it leave the application to a website that displays temperature, pollen or humidity levels.

The application would also benefit from more tracking and time sensitive capabilities, making it particularly beneficial in cases where patients are required to wait a certain period of time before taking next-steps. As they move down their PAAP checklist the timer could automatically start and keep track of this progression. Moreover, the addition of personalised notifications as an appropriate way to remind users of daily preventative medications. is a Finally, with significant development the PAAP smartphone application has the potential to be compatible with existing wearable devices which would allow for a greater accumulation of personal data in order to specifically assist the user in controlling their asthma

In the big picture, PAAPs as a smartphone application offers asthmatics an alternative way to control their asthma by giving them the knowledge, means and opportunity to do so in a technologically appropriate and convenient way. 


\section{CONCLUSION}

This paper looked into asthma management and ways to increase the use of Personalised Asthma Action Plans as an asthma intervention. The main aim of this research was to determine if the conversion from hand-written asthma action plans to a smartphone application, would improve their potential utilisation by asthmatics. It was hence hypothesised that Asthma action plans are more likely to be used when accessible via a smartphone application due to societies increased convenience and reliance on mobile devices.

Based on primary research and an extensive review of related work the crossover point of the IoT, PAAPs and smartphone applications was deemed an appropriate solution to address the underutilisation of PAAPs.

The resulting creation of a high-fidelity prototype where features of importance as determined by patients were incorporated into the application, in order to further enhance user experience and encourage increased use of PAAP smartphone application. In particular, the apps ability to access information regarding temperature, humidity and pollen levels within the environment gives asthmatics more personalised control over their asthma.

Consequently, results from primary research showing that over $80 \%$ of patients would use the PAAP smartphone application and believe that the change from a written to digital platform would increase their use of PAAPs as it is more convenient. This shows the original hypothesis to be true, making the PAAP smartphone application a step in the right direction for the future of asthma management and control.
With the technology of today, this integration and digital upgrade is a feasible and appropriate way to improve individual knowledge about asthma management and hence improve the utilisation of asthma action plans, as an asthma intervention.

\section{REFERENCES}

[1] Asthma Australia, 2017

[2] Australian Institure of Health, 2016

[3] National Asthma Council Australia, 2016

US Department of Health \& Human Services - National heart, lunch and blood institute, 2014

[4] Woolcock Institute of Medical Research, 2017

[5] Lynch, S., Kearney, S., Hueppe, M., Day, T., Dominey, R. \& Dewey, A. 2016, 'Owning my asthma: an enhanced self-management initiative for 14-19 year olds', npj Primary Care Respiratory Medicine, vol. 26, no. 16077.

[6] Engel, T., Rizvanolli, L., Pidgeon, H. \& Palter, J. 2017, '413 Incorporation of a Novel Asthma Action Plan to Improve Knowledge and Symptom Management in the Low Acuity Asthmatics Presenting to the Emergency Department', Annals of Emergency Medicine, vol. 70, no. 4, pp. S162.

[7] Joshua S Davis, Maoyun Sun, Alvin T Kho, Kip G Moore, Jody M Sylvia, Scott T Weiss, Quan Lu \& Kelan G Tantisira 2017, 'Circulating microRNAs and association with methacholine PC20 in the Childhood Asthma Management Program (CAMP) cohort', PLoS One, vol. 12, no. 7, pp. e0180329.

[8] Huckvale, K., Morrison, C., Ouyang, J., Ghaghda, A. \& Car, J. 2015, 'The evolution of mobile apps for asthma: an updated systematic assessment of content and tools', BMC medicine, vol. 13, no. 1, pp. 58.

[9] Jaimini, U. 2017, 'PhD Forum: Multimodal IoT and EMR Based Smart Health Application for Asthma Management in Children', IEEE, pp. 1-2.

[10] Sheth, A., Jaimini, U., Thirunarayan, K. \& Banerjee, T. 2017, 'Augmented personalized health: How smart data with IoTs and AI is about to change healthcare', IEEE, pp. 1-6. 